\title{
Valproate and epilepsy: for women as well as men
}

\section{Charlotte Lawthom}

\section{Correspondence to \\ Dr Charlotte Lawthom, Neurology, Aneurin Bevan University Health Board, Newport NP11 5GH, UK; charlotte.lawthom2@wales. nhs.uk}

Accepted 11 March 2018 Published Online First 23 April 2018

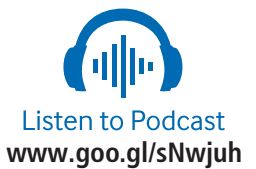

\section{SLinked}

- http://dx.doi.org/10.1136/ practneurol-2018-001932

Check for updates

To cite: Lawthom C. Pract Neurol 2018;18:222223.

\begin{abstract}
Sodium valproate remains the best drug for idiopathic generalised epilepsy. For men with the latter diagnosis, this is the drug of choice. Sodium valproate has an unacceptably high level of major fetal malformation and also causes learning disabilities in many children exposed to the drug in utero. Women of reproductive age should not normally be offered this drug. There are many women with refractory epilepsy who would benefit from this drug and who are not planning pregnancy. Individualised epilepsy care is the gold standard, not blanket bans on drug choice based on gender.
\end{abstract}

Gender equality, or rather the lack of it, is a pressing issue of our time. Epilepsy, in contrast, is a great respecter of parity between the sexes. While there are differences in epilepsy subtypes, there are no differences overall in epilepsy rates between the genders. ${ }^{1}$ Nevertheless, management guidelines for adults with epilepsy treat women differently. ${ }^{2}$ For the entirety of a woman's potential reproductive epoch, women are preferentially treated with antiepileptic drugs that are known to be safer for the fetus. ${ }^{2}$

Women planning a pregnancy are enormously relieved to have good quality data that offer reassurance around the exposure of their unborn baby to antiepileptic drugs. ${ }^{3}$ Routinely discussing pregnancy planning has become the gold standard in every epilepsy consultation with women of reproductive age. ${ }^{2}$ It is difficult to quantify the improvement that this focus on pregnancy in epilepsy has had for children born to mothers taking antiepileptic drugs. We want women to have healthy children and therefore advise them accordingly. This example of good practice has been a maxim for all epilepsy health professionals, certainly since I began practising medicine in the mid-1990s. The data from the UK pregnancy register, which has been repeatedly updated, have allowed us to have confidence in the information we provide. ${ }^{3}$ It is noteworthy though that the potential for pregnancy has now become a dominant consideration when treating women aged 15-45 years with epilepsy. The UK National Institute for Health and Care Excellence offers ample guidance around pregnancy and childbirth. However, women who do not become pregnant are mentioned only with respect to contraception and briefly, the menopause. ${ }^{2}$

Thus, all women of reproductive age risk being managed as though a pregnancy is imminent. Physicians are typically cautious, with the central tenet of 'first do no harm' embedded into our practice. In managing a woman of childbearing age with epilepsy, the emphasis is being put on potential risk of harm to the theoretical unborn child. This potential risk of harm must be balanced against the risk of harm to our female patient with epilepsy; she is not theoretical but current and contemporary. The risks she faces range from the poorly controlled seizures with their impact on everyday life, work and driving, through to injury and death.

Avoidable harm is a hugely emotive subject. Women planning pregnancy typically place greater weight on the health of the planned baby than their own epilepsy management. This emotive situation sometimes manifests as women failing to take antiepileptic drugs during pregnancy, or minimising the impact of their seizures in an attempt to avoid epilepsy drug escalation.

The UK media has recently made much of the teratogenic risks of valproate, placing the focus almost entirely on the child. The combination of the high rate of major fetal malformations that occur with valproate, along with a lower measurable IQ in $40 \%$ of children exposed to valproate in utero, has led to claims that 'valproate is the new thalidomide.' 
Neurologists have been prescribing valproate with great caution to women with epilepsy for decades. The UK's Medicines and Healthcare products Regulatory Agency (MHRA) has developed up to date guidance around valproate to reduce further any unnecessary risk to an unborn child. ${ }^{4}$

Interestingly, women in the developed nations are not bearing children as often as previously; in Europe, conception rates are falling precipitously. In the UK, migration is maintaining the overall birth rates, although conception rates per woman have fallen overall since the 1970s. ${ }^{5}$ Falling fertility in the developed world is ill understood and largely ignored, and the overall attribution of fertility to the falling birth rate is unclear. In 2016, the USA reported a record low fertility rate of 62 births per 1000 women aged 15-44 years. ${ }^{6}$ What seems clear however is that increasing numbers of women and couples are making considered and deliberate choices not to have children. According to the 2014 US Census Bureau's Current Population Survey, $47.6 \%$ of women aged $15-44$ years had never had children-the highest percentage of childless women since tracking began in $1976 .{ }^{6}$

Among women who opt to have a child, many are delaying that decision, and often have only one child. The UK data around birth rates by maternal age are interesting. The conception rate for women aged 40 and over has more than doubled since 1990, with similar increases in women aged 35-39 years. Women aged 30-34 years have continued to have more children, with continuous rises since 1990. At the younger age range, teenage pregnancies have continued to drop to their lowest levels since records began in $1969 .^{5}$

Increasingly, therefore, women spend the vast majority of their lives neither pregnant nor wishing to conceive. There is no reason to think that women with epilepsy fall outside this modern demographic.

Epilepsy is associated with an increased mortality, and avoidable deaths from epilepsy have not fallen. The risk of death from sudden unexpected death

\section{Key points}

Epilepsy care should be equal across gender.

- Most women are not pregnant most of the time.

- Pregnancy in epilepsy should be planned.

- Women should receive access to all drugs and receive all information. in epilepsy (SUDEP) increases with greater seizure frequency. Seizure control is literally a matter of life and death. To date, valproate remains the best treatment for idiopathic (genetic) generalised epilepsies. ${ }^{7}$

Women, like men, need a tailored approach to their epilepsy management. Women must have equal access to all treatments for epilepsy. We, as health professionals, must ensure that we provide the best care for our patients. Women with epilepsy deserve clear comprehensive information about the risks to themselves as well as to any unborn and potential child. Women who do not plan to become pregnant must be afforded the same opportunities to treat their epilepsy as men. All women deserve all available epilepsy treatment options and must receive treatments according to need, and not to our notions around gender and reproduction.

Contributors The idea for submitting opposing views arose following presentations by John Craig and CL.

Competing interests CL has received speaker fees and consultancy fees from UCB Pharma and Eisai.

Patient consent Not required.

Provenance and peer review Commissioned; externally peer reviewed by Sanjay Sisodiya, London, UK.

(C) Article author(s) (or their employer(s) unless otherwise stated in the text of the article) 2018. All rights reserved. No commercial use is permitted unless otherwise expressly granted.

\section{REFERENCES}

1 Christensen J, Kjeldsen MJ, Andersen H, et al. Gender differences in epilepsy. Epilepsia 2005;46:956-60.

2 Nice. Epilepsies: diagnosis and management Clinical guideline [CG137], 2012.

3 Campbell E, Kennedy F, Russell A, et al. Malformation risks of antiepileptic drug monotherapies in pregnancy: updated results from the UK and Ireland Epilepsy and Pregnancy Registers. $J$ Neurol Neurosurg Psychiatry 2014;85:1029-34.

4 Toolkit on the risks of valproate medicines in female patients. Medicines \& Healthcare products Regulatory Agency. https:// www.gov.uk/government/publications/toolkit-on-the-risks-ofvalproate-medicines-in-female-patients

5 Office of National Statistics. Statistical bulletin: conceptions in England and Wales: 2015 Annual statistics on conceptions covering conception counts and rates, by age group including women under 18 .

6 U.S. Census Bureau. Population. https://census.gov/topics/ population.html

7 Marson AG, Al-Kharusi AM, Alwaidh M, et al. The SANAD study of effectiveness of valproate, lamotrigine, or topiramate for generalised and unclassifiable epilepsy: an unblinded randomised controlled trial. Lancet 2007;369:1016-26. 